\title{
L'HYDROLOGIE DU RHÔNE, LES AMÉNAGEMENTS DU CHENAL ET LA GESTION TERRITORIALE DE SES PLAINES EN AVAL D’ORANGE
}

\author{
Philippe LeveAu
}

Mots-clés. Bas-Rhône, cadastres d'Orange, canaux, céréaliculture, fleuve, frontière, inondation, marais, ponts, urbanisme.

Key-words. Lower Rhône, the Orange cadastre, channels, cereal culture, river, frontier, flood, marsh, bridges, urbanism.

Résumé. Sur la section en aval d'Orange, deux articles ont été consacrés à Avignon et Arles. Sont donc présentés ici le chenal et le lit majeur du fleuve dans ses sections rurales, ses franchissements et les aménagements du chenal suscités par ses difficultés d'écoulement (la fossa Augusta de la plaine d'Orange). Ainsi cette histoire permet d'observer les changements apportés par Rome. Les incidences de l'hydrologie du fleuve sur l'occupation de la plaine d'Orange, de la plaine d'Arles et de la Camargue sont traitées à partir des nouveautés fournies par les recherches pluridisciplinaires conduites sur la vallée des Baux. Dans le domaine territorial, Arles et Avignon s'affirment comme villes du fleuve.

\begin{abstract}
Two projects are presented here that have been undertaken on the section downstream from Orange, at Avignon and Arles. The main course of the river, along with its crossings, and the modifications undertaken on the channel which were necessitated by discharge problems (the fossa Augusta on the Orange plain) are considered. These developments allow us to consider the changes brought about by Rome. The impact of the river's hydrology on settlement on the Orange plain, as well as that around Arles, and also on the Camargue, are considered from a multidisciplinary point of view, exploiting research carried out in the vallée des Baux. Within the territorial context, both Arles and Avignon affirm themselves as river-towns.
\end{abstract}

En aval de la plaine d'Orange, le couloir rhodanien s'élargit. À une série de bassins séparés par des défilés succède une plaine qui atteint $45 \mathrm{~km}$ d'est en ouest au niveau de Carpentras et jusqu'à $60 \mathrm{~km}$ à hauteur d'Arles (fig. 39) où commence la zone deltaïque. Depuis le confluent avec la Drôme, au sud de Valence, le fleuve, exogène par apport à la zone climatique dans laquelle il pénètre, voit son hydrologie progressivement modifiée par ses affluents méditerranéens. Du tableau des conditions hydrologiques durant le millénaire qui va du début du dernier Âge du Fer jusqu'à la fin de l'Antiquité $\left(V^{e} s\right.$. avant J.-C./ $\mathrm{V}^{\mathrm{c}} \mathrm{s}$. après J.-C.), nous retiendrons que « l'activité hydrosédimentaire est irrégulière, mais elle n'induit pas de transformations majeures des lits fluviaux. Ses fluctuations sont mieux perçues en milieu deltaïque où la faible dénivellation de la plaine permet une meilleure expression des crues : les débordements y sont plus fréquents et les taux de sédimentation plus significatifs que sur les sites urbains. " (cf. Provansal et al., supra, p. 25-28). La période se termine par « une recrudescence des apports liquides exceptionnels et des apports solides, aboutissant à une métamorphose des milieux 


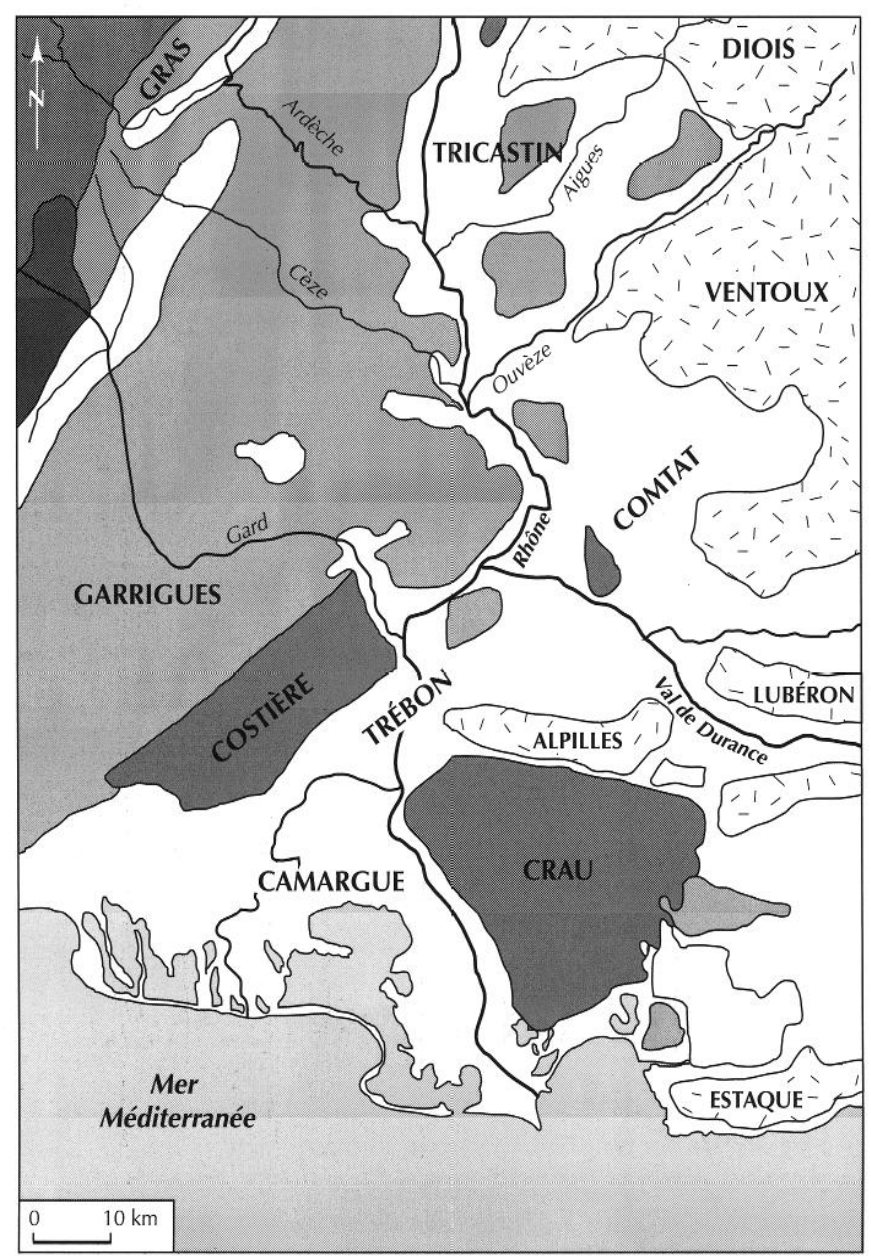

Fig. 39 - Croquis de la zone étudiée (d'après Béthemont, 1972, pl. 1).

fluviaux »; renouant avec les conditions qui prévalaient au Premier Âge du Fer, elle dure jusqu'au VIII ${ }^{e}$ s. environ.

Le Rhône traverse le secteur de la Gaule où la présence romaine se manifeste par la plus forte densité de villes. Deux d'entre elles, Arles et Orange, sont des colonies de vétérans; la première, citée par Strabon comme $\pi \circ \lambda \iota \sigma \kappa \alpha t \varepsilon \mu \pi o \rho t o v$ ov $\mu \imath \chi \rho \circ v$ (ville et centre commercial, non négligeable), est le port du Rhône pour sa partie aval (Strabon, IV, 1, 6). La romanisation des campagnes n'est pas moins forte; elle s'est en particulier traduite par la mise en place d'une trame géométrique de centuriations sur laquelle les marbres d'Orange donnent de grandes précisions. Nous insisterons sur les nouveautés qu'elle introduit dans l'organisation des paysages fluviaux et la gestion territoriale.

\section{LES AMÉNAGEMENTS DU CHENAL EN AVAL D'ORANGE}

Les conditions générales d'écoulement du fleuve sont, pour l'Antiquité, proches de celles d'aujourd'hui : une pente qui reste forte $(0,48 \mathrm{~m}$ au kilomètre entre Ardèche et Durance, 0,25 m entre Aramon et Beaucaire) et deux obstacles (géologiques) susceptibles de ralentir l'écoulement, le Roque d'Acier au droit d'Aramon, et, dans le delta, le Seuil de Terrin. Mais la stabilité du contexte géologique n'empêche pas le Rhône antique d'avoir eu un chenal sensiblement différent de celui qu'il avait avant les aménagements modernes. Les difficultés d'écoulement sont en effet aggravées dans le delta par d'anciens cordons littoraux générateurs de méandres et, en amont, par des accumulations sédimentaires formant bouchons aux confluents des affluents méditerranéens qui développent des cônes dont la variabilité est conditionnée par leur régime (Béthemont, 1972, p. 90 et Provansal et al., supra, p. 25-28).

L'inégal avancement des recherches sur l'hydrologie fluviale, la géomorphologie du chenal (ou des chenaux) et sur l'histoire des aménagements du fleuve explique le déséquilibre de la présentation qui suit. La plaine d'Arles et le secteur deltaique sont mieux connus que les sections amont.

\section{LES CONDITIONS OFFERTES À LA NAVIGATION ET LES FRANCHISSEMENTS}

Actuellement, le Rhône mesure $180 \mathrm{~m}$ au sud de l'Ardèche, $190 \mathrm{~m}$ au sud d'Avignon et jusqu'à $250 \mathrm{~m}$ à Fourques. Mais pas plus que dans son cours amont, cet obstacle n'a jamais constitué une véritable coupure entre les deux sections de plaine qui se font face. La relation entre les deux rives était assurée par une batellerie dont l'importance est attestée par deux témoignages relatifs à des franchissements du Rhône par des armées. Le premier est le récit de la traversée du sud de la Gaule par Hannibal qui, en 218, tente d'envahir l'Italie depuis l'Espagne (Polybe, III, II, 43; Tite-Live, XXI, 28). Le second est un épisode de l'offensive du chef arverne Bituit qui, en 121 avant J.-C., franchit le fleuve sur deux ponts. L'un existait, l'autre est un pont de bateaux dont la rupture aggrava le désastre gaulois (Strabon, IV, 1, 11 ; 2, 3; Orose, V, 14, 3). Ces deux récits font l'objet de 
débats. Les points de franchissement ne sont pas assurés. Orose situe la traversée de Bituit au confluent avec une rivière appelée Isara, dont il n'est pas assuré qu'elle soit notre Isère. Ce témoignage a été discuté par C. Jullian pour qui il n'aurait pu trouver là assez de bateaux pour doubler le pont existant. En revanche, le témoignage de Polybe sur la batellerie dans la partie aval n'est pas contesté.

Des argumentations de nature historique et environnementale sont utilisées conjointement. Invoquant un état (récent) du chenal fluvial, C. Jullian déplace vers le sud les franchissements par Hannibal et par Bituit : Hannibal serait passé par Tarascon/Beaucaire (Jullian, 1920-1926, rééd. 1993, p. 942, note 78) et Bituit vers Bollène et même Orange (Jullian, ibid., p. 1035, note 46). Pour cet auteur, la confluence Isère/Rhône ne paraît pas un lieu propice au pont fixe utilisé par Bituit. De même, M. Clerc situe près du confluent de la Durance, dans la Montagnette plutôt qu'au confluent de l'Isère, l'emplacement du camp où, entre 104 et 102, Marius vient attendre les barbares (Clerc, 1906). En fait ces épisodes et, d'une manière générale, les récits d'opérations militaires prouvent l'utilisation de la voie d'eau pour la remontée et la descente de la vallée. Scipion qui tente d'atteindre Hannibal (Polybe, 45, 4) l'utilise pour le transport de ses bagages. Marius s'installe au bord du Rhône pour faciliter son ravitaillement. D'autre part, les progrès accomplis dans la connaissance de l'histoire du chenal permettent une relecture des sources écrites relatives à ces épisodes, en particulier à celui de la traversée d'Hannibal ${ }^{76}$.

Le seul pont sur la matérialité duquel nous disposons de détails est le pont romain d'Arles où le dédoublement du chenal constituait un facteur favorable à l'installation d'un pont (cf. Arcelin et al., infra, p. 128). Qu'en était-il en amont? Le bilan de l'archéologie avignonnaise réalisé par D. Carru ne fait état d'aucun vestige certain de pont, alors même que les découvertes effectuées sur la rive droite attestent l'étroitesse des relations entre les deux rives ( $c f$. Carru, infra, p. 117). Entre Avignon et Arles, les itinéraires et les vestiges routiers s'accordent à placer la traversée du Rhône par la voie domitienne, le Trajectum Rhodani, entre Beaucaire/Ugernum et Tarascon/Tarusco (Michelozzi,

76. Cette relecture a dû être supprimée pour alléger l'exposé. Elle sera publiée ultérieurement.
1997). Mais aucune trace matérielle n'en a jamais été trouvée. Elle se faisait par bacs, selon des procédés décrits par C. Jung et T. Odiot, pour la partie du Rhône située plus en amont.

Une attention insuffisante portée à l'archéologie du fleuve explique les lacunes de ce « dossier ». Les résultats positifs des travaux réalisés à Arles par les plongeurs du DRASM le démontrent (Long, 1994). Mais il faut souligner ici les facteurs liés à l'activité du fleuve lui-même. L'anecdote de la traversée du Rhône par Hannibal témoigne d'un état du chenal totalement différent de celui du Rhône figuré sur la carte de Cassini. De même, une étude critique des nombreuses découvertes, effectuées à l'occasion de la construction de la centrale hydroélectrique d'Aramon, montre qu'il s'agit souvent de matériaux antiques jetés dans le fleuve ou chargés dans des bateaux coulés pour endiguer des bras du fleuve aux $\mathrm{XVIII}^{\mathrm{e}}$ et XVIII ${ }^{\mathrm{e}}$ s., comme on le sait pour Vallabrègues (Leveau, à paraître b).

\section{LES AMÉNAGEMENTS DU RHÔNE : LES FOSSAE MARIANAE ET LA FOSSA AUGUSTA D'ORANGE}

Dès les premiers temps de la conquête romaine. (fin de l'Âge du Fer), la navigation a donné lieu à des aménagements considérables. Un article de ce « dossier » est consacré au canal de Marius et à sa relation avec les dynamiques fluviale et littorale. Cet article a été également l'occasion de présenter l'état des recherches sur les embouchures du fleuve ( $c f$. Vella et al., infra, p. 131-139).

Le canal de Marius n'est pas la seule réalisation romaine. Le cadastre $\mathrm{C}$ d'Orange montre que l'aménagement $d u$ chenal fluvial à la navigation fut poursuivi ver's l'amont. Ces dernières années, cette centuriation avait été recherchée en Camargue puis dans la région de Valence, là où le Rhône butte sur le Massif central, développant un marécage en rive droite. M. Christol, J.-C. Leyraud et J.-C. Meffre viennent de montrer qu'elle devait bien être placée dans la plaine d'Orange, comme le pensait déjà A. Piganiol (Christol et al., 1998). Cette proposition paraît rencontrer l'adhésion des spécialistes. Il reste cependant à valider le positionnement des fragments de ce cadastre : d'autres possibilités existent (Chouquer, comm. orale) (fig. 40). 


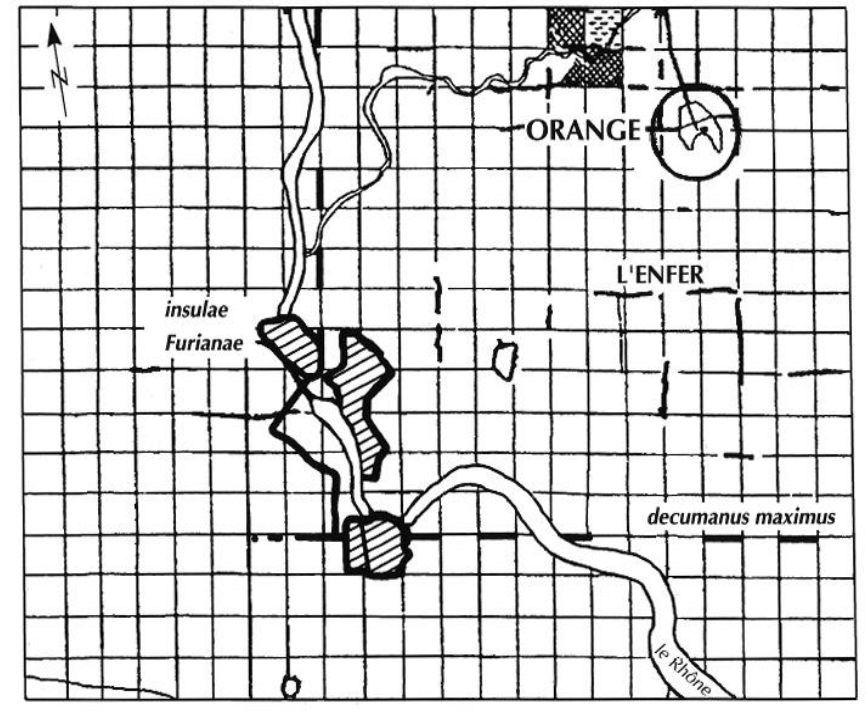

Fig. 40 - Les insulae Furianae et la fossa (d'après Ballais, Meffre, 1997, p. 315, fig. 4).

F. Salviat avait mis en évidence le rôle d'une fossa Augusta, large de $30 \mathrm{~m}$, figurant sur le fragment 351 . Ce n'est pas un canal de drainage mais un canal de navigation (Salviat, 1986). Les travaux de J.-L. Ballais et de J.-C. Meffre en précisent maintenant la fonction : permettre à la navigation de contourner une zone où le cours du Rhône était encombré d'îles, la rendant difficile. De tels aménagements ont des parallèles sur le Danube : la Mutatio fossis en Pannonie inférieure, entre le Danube et la Save, garde le « souvenir d'un canal qui drainait les eaux de cette région très marécageuse et évitait à la navigation la boucle de la Save " (Constans, 1921, p. 198) ${ }^{77}$. Sur le même fleuve, Trajan avait aménagé les Portes-de-Fer en faisant creuser un canal connu par une célèbre inscription (Sasel, 1973). Comme le canal de Marius dans la zone orientale du delta du Rhône, la fossa d'Orange dut avoir une incidence sur le drainage de la plaine, soit sur les fameuses zones humides, mais ce n'était pas l'objectif de son creusement.

Les travaux de J.-L. Ballais et de J.-C. Meffre éclairent en outre certaines caractéristiques du chenal fluvial

77. Il existe sur le Danube un autre canal dans le secteur des Portes-deFer, $c f$. J. Sasel, Trajan's canal at the Iron Gate, Journal of Roman Studies, 1973, p. 80 et D. Boscovic, Aperçu sommaire sur les recherches archéologiques du limes romain et paléobyzantin des Portes-de-Fer, MEFRA, $1978,90,1$, p. 431. antique. F. Salviat a identifié l'historien d'Alexandre, Q. Curtius, comme l'aménageur - l'inventor-d'un archipel du Rhône, les insulae Furianae, dans le cadre d'opérations de lotissement au début de notre ère. L'interprétation de sondages effectués par la CNR les conduit à rechercher ces îles à l'ouest de Caderousse. En un point du fleuve, caractérisé par l'existence d'un seuil et l'apport d'affluents, des îles se font et se défont. Recouvrant les galets d'une nappe würmienne, le fleuve dépose des limons et crée un terroir riche pour qui en maîtrise l'hydraulique (Ballais, Meffre, 1997, p. 312 et 313, fig. 2 et 3). Il n'est pas surprenant qu'aucune trace n'en ait été trouvée : le fleuve peut aussi reprendre ce qu'il a donné. Pour le secteur d'Aramon entre 1813 et 1885 , J. Béthemont en donne une illustration graphique et un commentaire bien propre à nous dissuader de rechercher sur les photos aériennes la trace de l'archipel perdu. "L'île de Mouton s'élevait au début du $\mathrm{XIX}^{\mathrm{e}}$ siècle, sur la commune d'Aramon, un peu en amont de l'ancien confluent de la Durance. Propriété seigneuriale, elle fut partagée entre les habitants en 1793, par lots égaux de 3 ares, qui furent plantés de vignes. Mais en 1813, l'île disparut à la suite de travaux modifiant le site de confluence. Or, dans les années suivantes, une île nouvelle, dite de Casseyrolles, se forma un peu en aval et se déplaça lentement, jusqu'à occuper l'emplacement de l'ancienne île de Mouton " (Béthemont, 1972, p. 95, fig. 29 et 140).

\section{HYDROLOGIE ET OCCUPATION DES SOLS DANS LE LIT MAJEUR DU RHÔNE}

Au sud d'Orange, le couloir rhodanien s'élargit jusqu'à la zone deltaiqque où le lit majeur du fleuve connaît son extension maximale. Définie par le champ d'inondation de la crue de mai-juin 1856, cette zone où le marais se développe de manière préférentielle inclut actuellement des bassins hydrographiques affluents creusés sous le 0 NGF et dont le fond se situe sous le remblaiement alluvial récent (en rive gauche, les marais de basse Ardèche, de la Cèze et du Gardon inférieur; en rive droite, ceux de La Palud, d'Orange, de Sorgues et de Saint-Rémy). Large de $5 \mathrm{~km}$ dans les plaines d'Avignon et de Vallabrègues, elle atteint $12 \mathrm{~km}$ à Tarascon. Au sud de Beaucaire, elle s'élargit entre les plateaux de Crau et 


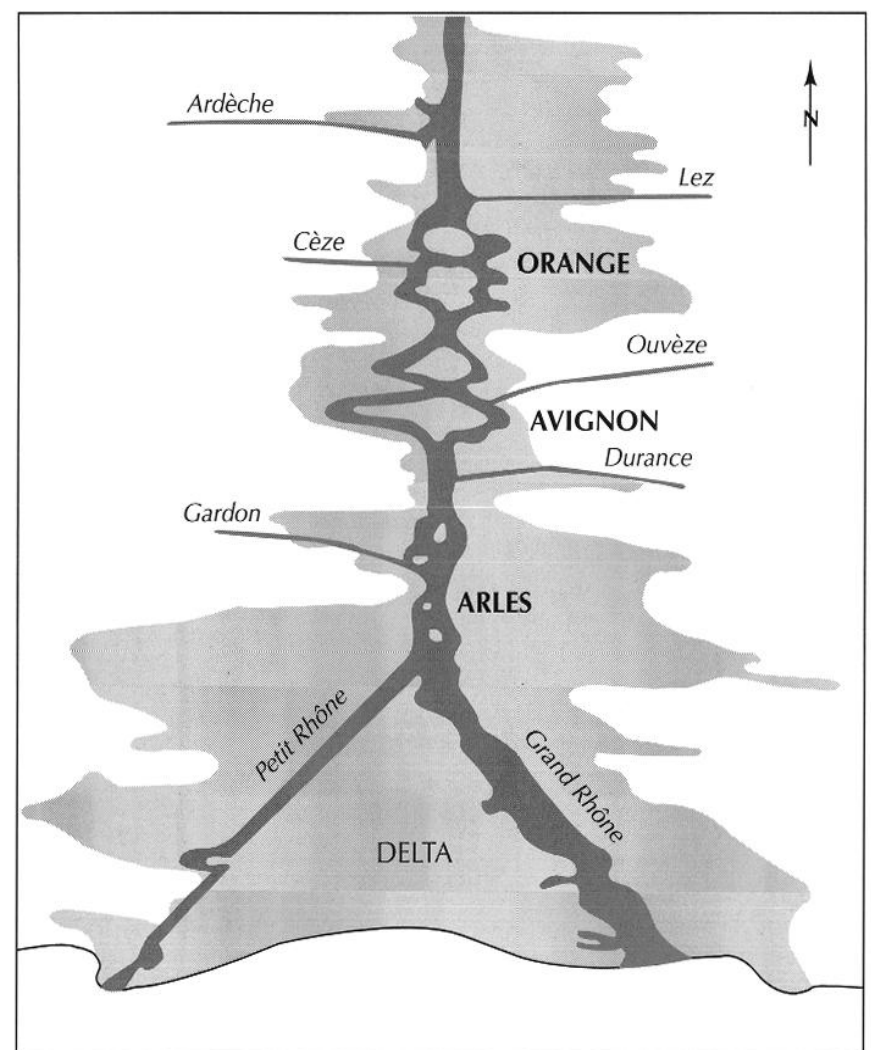

Fig. 41 - La plaine d'inondation du Rhône définie d'après l'extension de la crue de 1856 (d'après le Service de la navigation Rhône-Saône et d'après Arnaud-Fassetta, 1998, p. 9, fig 3).

Costière, englobant quelques surfaces distinctes de la vallée comme le marais des Baux et la région comprise entre Fos et le Galejon. En aval d'Arles, dans le delta, le lit majeur se développe sur 30 à $40 \mathrm{~km}$ (fig. 41).

Les recherches en cours permettent d'ébaucher une histoire de ce lit majeur. Dans l'attente de la publication des fouilles occasionnées par la construction de la ligne 5 du TGV, les premiers résultats seront évoqués. Mais l'essentiel de l'exposé s'appuie encore sur les recherches réalisées par les équipes aixoises.

\section{D'ORANGE À TARASCON}

Pour le secteur de rive droite s'étendant au sud du confluent avec l'Ardèche, d'importants résultats sont attendus des fouilles réalisées à l'occasion du long transect ouvert pour la ligne $5 \mathrm{du}$ TGV dans les vallées de Cèze et de la Tave, secteur inclus dans la zone d'extension du cadastre B d'Orange. Cette opération offre l'oc- casion de dépasser l'obstacle à une vision synthétique de l'évolution de la plaine que constitue la fonction actuelle de limite jouée par le cours du Rhône entre les départements du Gard et du Vaucluse qui relèvent de deux régions (administratives). En face, dans la plaine d'Orange, les fouilles ont montré que les sites d'époque romaine, situés dans l'actuel lit majeur, étaient recouverts par 3 à 4 m d'alluvions (Brochier, 1997, p. 95; cf. Provansal et al., supra, p. 22). Seuls sont donc visibles en prospection les sites situés en position haute ; cette observation permet de relativiser les cartes archéologiques de même qu'elle pose le problème de l'état de l'hydrologie antique de ce secteur.

En rive gauche du Rhône (Vaucluse), dans l'attente de la publication de ces fouilles, qui ont accordé une place considérable aux approches environnementales, on dispose déjà des premiers résultats des travaux de J.-L. Ballais et J.-C. Meffre sur Orange. Depuis la fin du Moyen Âge, lors de crues majeures, la région d'Orange est dans le lit majeur de l'Aygues, ce qui a justifié des endiguements. Le lit mineur de cette rivière paraît stable durant la période historique, mais, à partir de la fin de l'Antiquité, ses inondations semblent menacer la rive gauche (Meffre, Ballais, 1996, p. 70 et 76). Auparavant déjà, la zone était inondée par une rivière qui n'est vraisemblablement pas l'Aygues mais son affluent, la Meyne. C'est ce qui ressort de la confrontation des fragments des marbres du cadastre $\mathrm{B}$ et des données sédimentologiques attestant une crue à l'époque augustéenne (Mignon, 1996 , p. 219).

En rive droite du Rhône (gardoise), l'attention s'était portée surtout sur les sites de hauteur de Gaujac (Provost, 1999) et Laudun (id., ibid.). Pourtant, en contrebas, en bordure du fleuve, la construction de la centrale nucléaire de Marcoule a entraîné la destruction d'un site sans doute portuaire (id., ibid.). M. Christol et J.-L. Fiches citent plusieurs installations gardoises en relation avec le commerce du Rhône ( $c f$. infra, p. 147). Prospections archéologiques et fouilles liées aux travaux de construction de la ligne du TGV ont attiré l'attention sur l'ancienneté de la maîtrise du milieu. À l'époque gallo-romaine, la villa de La Ramière, proche de Roquemaure, domine une plaine d'inondation dans laquelle aucun site antique n'est connu. Au franchissement de la Tave, l'identification de fossés qui remonteraient à l'Âge du Bronze prouve la précocité de l'occupation agricole de ces zones humides. 


\section{LA PLAINE D'ARLES}

L'image de l'hydrologie de la plaine d'Arles dans l'Antiquité a été renouvelée par les travaux pluridisciplinaires (archéologie, géomorphologie, palynologie) conduits depuis maintenant une dizaine d'années dans la vallée des Baux. Délimitée par la courbe de niveau des $20 \mathrm{~m}$, cette dépression de 1 à $2 \mathrm{~km}$ de large qui sépare les Alpilles de la Crau sur $12 \mathrm{~km}$ d'est en ouest constitue un excellent observatoire des phénomènes hydrologiques régionaux. Des études sédimentologiques, conduites par M. Jorda et M. Provansal sur les sondages archéologiques effectués en 1990 au pied des moulins romains de Barbegal, ont remis en question l'image d'une inondation permanente de cette dépression, dont le fond se situe à peine au-dessus du niveau de la mer (Leveau, 1996, 1997). En 1995, une campagne de forages a eu lieu. En 1996, la pose d'un gazoduc a entrainé la découverte d'un établissement (village?) remontant au Chalcolithique, apportant une confirmation spectaculaire des hypothèses proposées. Des dépôts correspondant aux différents fonctionnements hydrauliques de la vallée ont été mis en relation avec la documentation écrite et les données archéologiques. Il est désormais possible d'y suivre la montée des eaux durant l'Holocène. Le mouvement n'est pas linéaire; il se décompose en une succession de phases d'avancées, de pauses et de régressions en relation avec la variabilité climatique. La phase d'inondation, qui affecta la vallée à la fin du Moyen Âge et aux débuts des Temps modernes, est probablement en relation avec l'hydrologie du "Petit Âge glaciaire ». Cependant, facteurs anthropiques et climatiques se combinent. La tendance à l'inondation fut accentuée par l'édification d'un barrage pour éviter que, lors des grandes pluies méditerranéennes tombant sur les Alpilles, le marais de Barbegal ne déborde vers la plaine d'Arles. L'actuel assèchement résulte d'un siècle de travaux conclus par la transformation de la dépression en polder continental. L'extension de la crue de 1856 montre en effet l'intégration de la vallée des Baux dans le lit majeur du Rhône

À l'époque romaine, la situation était très différente de celle qu'avait imaginée F. Benoit à partir du milieu amphibie médiéval et moderne. Influencé par la doctrine des dessiccateurs dans son interprétation des moulins de Barbegal, il avait cru à une liaison par eau entre l'usine et Arles : au pied du chainnon aurait été aménagé un chenal emprunté par les radeaux chargés de blé (Benoit, 1940a). Image tenace héritée des ingénieurs de l'époque romantique, elle reste prégnante. La maquette récente de l'usine réalisée pour le musée de l'Arles antique l'a reprise, alors que la période romaine est, au contraire, caractérisée par un abaissement du plan d'eau. À une époque où la vallée des Baux n'est pas atteinte par les crues du Rhône, elle a même sans doute fait l'objet d'un drainage (Bruneton et al., à paraître).

Son horizontalité actuelle résulte en effet d'apports sédimentaires colmatant une topographie dont les forages ont montré la diversité (au moins deux cuvettes) (Bruneton et al., 1998). Tout à fait analogue à celui qui a été mis en évidence plus au nord, dans la plaine d'Orange et ailleurs, ce colmatage rend compte de la carte archéologique telle qu'elle ressort des prospections réalisées par M. Gazenbeek (Gazenbeek et al., 1996; Gazenbeek, à paraître). Les traces d'une occupation antique de la vallée n'avaient pas été reconnues, tout simplement parce que la permanence supposée de l'inondation la rendait invraisemblable. Il apparaît maintenant que les moulins n'étaient pas isolés : à proximité existait une importante villa appartenant à une riche famille arlésienne. Elle comportait une partie résidentielle ; une de ses annexes entrait dans la série des entrepôts à dolia des villae viticoles connus en Provence. Au vallon des Arcs, des élément démontés du mausolée familial ont servi aux dernières restaurations du pont de l'aqueduc d'Arles (Gascou, Leveau, 1998). Par la suite, contrairement à ce que l'on écrivait depuis F. Benoit, l'occupation humaine ne s'était pas précocement repliée vers le nord, abandonnant le fond de la vallée devant la montée de l'eau : la vallée est restée occupée comme l'ont montré les fouilles réalisées sur un cimetière daté des $\mathrm{VII}^{\mathrm{e}}-\mathrm{VIII}^{\mathrm{c}} \mathrm{s}$. (Bellamy, Hitchner, 1996).

Il n'est évidemment pas question de nier l'importance de l'eau et la déprise agricole dans le paysage médiéval de la région. L'abbaye de Montmajour émerge comme une île au milieu des marais, au maintien desquels les moines sont attachés (Stouff, 1993). L'étude des sédiments dans la vallée permet de suivre la reconquête végétale du Haut Moyen Âge mise en évidence à partir des forages pratiqués (Andrieu et al., à paraître ; Riera Mora, à paraître). Mais cette évolution ne peut pas être généralisée à la plaine d'Arles. Les fouilles du gazoduc ont amené la découverte d'un moulin à turbine creusé dans un banc de rocher au bord du marais d'Arles, à La 
Calade. Daté de la fin de l'Antiquité, ce moulin était mu par un courant d'eau issu probablement de l'ancien aqueduc romain (Amouric et al., à paraître). L'installation, qui fut abandonnée à la suite de la montée du plan d'eau, prouve la persistance d'une économie domaniale sur laquelle on ne disposait que du témoignage très indirect des sarcophages de l'aristocratie arlésienne. Mais gardons-nous de généralisations hâtives! Le dépouillement de la colonne sédimentaire prélevée sur le site nous renseigne sur les rythmes de l'inondation dans cette partie de la plaine d'Arles ; à la différence de ceux de la vallée des Baux, les diagrammes polliniques n'enregistrent ici aucune reconquête végétale particulière, probablement du fait de la persistance de l'économie agricole.

Il n'existe encore aucune certitude sur la nature des cultures pratiquées. Cependant, les sols d'un marais drainé sont favorables aux cultures céréalières et les premiers résultats connus des analyses polliniques en cours paraissent confirmer la présence de ces cultures (Laval, Médus, 1994). Par ailleurs, l'activité agricole peut offrir une solution alternative aux hypothèses formulées quant à la mise en place d'une transhumance des moutons d'Arles à l'époque romaine. Les travaux de O. Badan, J.-P. Brun et $\mathrm{G}$. Congès ont révélé l'importance de l'élevage ovin dans la Crau toute proche : des bergeries en grand nombre suggèrent une exploitation systématique de cette plaine à l'époque romaine. Mettant cette activité en relation avec la transhumance, ils invoquent le parallèle de l'Italie méridionale où elle existe depuis le II $\mathrm{s}$. avant J.-C. (Badan et al., 1995). En l'absence de preuves archéologiques (mais que savait-on de l'élevage en Crau avant leurs prospections !) et compte tenu des objections techniques à de tels déplacements de troupeaux, il est possible que ceux qui séjournaient en Crau durant l'hiver aient trouvé, l'été, de quoi subsister dans les zones humides de la plaine du Rhône, en Camargue et dans la vallée des Baux (Columeau, à paraître).

\section{LA CAMARGUE}

La vallée des Baux ne couvre que quelques centaines d'hectares. La Camargue est la plaine deltaïque d'un des trois plus grands fleuves européens. Nonobstant cette différence d'échelle, les problèmes d'hydraulique et d'accumulation sédimentaire présentent d'évidentes analogies. D'autre part elle se trouve sur le territoire de la colonie d'Arles. C'est dire que l'on y retrouve les mêmes problèmes de mise en valeur!

Deux hypothèses, qui d'ailleurs ne sont nullement contradictoires, avaient cours. Selon la première qui remonte au siècle dernier, dès l'Antiquité, la Camargue dont le nom viendrait de celui d'un riche sénateur, Annius Camars -, et d'une manière générale l'ensemble de la basse plaine deltaíque auraient constitué une importante zone de production de blé, assise de la prospérité de la colonie romaine (CIL XII, 670 ; Pflaum, 1978, p. 312-313 ; Burnand, 1982, p. 412-413). Cette prospérité remonterait à l'époque de la colonisation grecque. D'après les sources antiques, les Grecs y auraient installé les colonies de Rhodanousia et Heraclea (Benoit, 1965, p. 132), qui, en même temps que des comptoirs, auraient été des centres de peuplement auxquels serait liée une vie agricole, sur le modèle de sites grecs coloniaux de cette époque. La seconde hypothèse est due à $\mathrm{F}$. Benoit ; sa vision d'un paysage et d'une économie dominés par le marais l'avait conduit à proposer une image très différente du delta : son rôle de "grenier de Rome " serait légendaire (Benoit, 1959 ; 1965, p. 116, note 33). De fait, en l'absence de données archéologiques certaines, les sources épigraphiques attestant la place d'Arles dans l'alimentation en blé de Rome étaient ambiguës : des blés transitant par le port d'Arles pouvaient fort bien y être arrivés par la voie rhodanienne. Cet auteur préférait donc mettre l'occupation humaine dans le delta en relation avec l'exploitation des ressources de la mer pour la pêche et le sel. Soulignons-en les implications! Arles aurait dû sa prospérité au contrôle du commerce fluvial (Benoit, 1965, p. 116). Il adhérait en effet à l'image que l'on avait de l'action de Rome telle que l'exprimaient C. Jullian (1920-1926, rééd. 1993, p. 443) et L.-A. Constans ; la création d'Arles obéissait à deux objectifs : abaisser Marseille et « la substituer à celle-ci dans son rôle de grand port méditerranéen " (Constans, 1921, p. 205). La colonie romaine aurait ainsi privé Marseille (grecque) des bases de sa richesse. En fait, les recherches récentes sur Marseille infirment cette théorie.

Le Service régional de l'archéologie de ProvenceAlpes-Côte d'Azur a développé un programme de prospections et de fouilles dont les premiers résultats confirment la réalité d'une occupation de la Camargue durant l'Âge du Fer (Arnaud-Fassetta, Landuré, 1997). Pour l'époque romaine, on y connaissait l'existence de 
domaines agricoles. Les fouilles en cours préciseront le statut des établissements reconnus et leur place dans l'habitat. Mais, d'ores et déjà, la relation entre carte archéologique et recouvrements sédimentaires apparaît avec évidence. Comme dans la plaine d'Orange, les recouvrements alluviaux expliquent que les cartes archéologiques ne reflètent pas l'importance de l'occupation du sol aux périodes anciennes. Inondations et dynamiques propres au delta conjuguent leurs effets. Dans sa partie sud, le delta est de formation moderne et, dans sa partie centrale, des tassements rendent invisibles les sites les plus anciens, de sorte que ceux qui ont été identifiés sont situés vers la tête du delta. Ainsi, la prise en compte de la dynamique deltaïque évite de confondre la " taphonomie " des sites avec un " front de colonisation " qui se serait développé depuis Arles, soit de rechercher une cause politique à une répartition des sites qui traduit un phénomène naturel. Un carottage effectué au bord nordest de l'étang du Vaccarès apporte un élément nouveau en montrant l'existence de champs cultivés, soit une production de céréales à une date postérieure à 3000 ( $c f$. Arnaud-Fassetta $e t$ al., à paraître). Bien que l'on ignore encore tout de la localisation des villages où auraient résidé les agriculteurs de la première moitié du premier millénaire avant J.-C., leur existence est hautement vraisemblable comme elle l'était dans la vallée des Baux avant les découvertes occasionnées par le creusement de la tranchée du gazoduc.

Les prospections ne permettent pas de vérifier ni d'infirmer l'hypothèse d'une colonisation par des agriculteurs venus de Grèce dès l'époque archaïque. Mais il est sûr qu'à l'époque romaine, le niveau marin se situait quelques décimètres au-dessous du niveau actuel et, comme dans les zones lagunaires languedociennes, le drainage naturel de la plaine était plus facile ; le cours dı fleuve était plus direct; le nombre des méandres était moindre que sur le Petit Rhône actuel et les alluvions étaient mieux évacuées (Arnaud-Fasseta, 1998). Quelle que soit l'importance des activités liées à la mer dans l'économie du delta (pêche, collecte du sel), l'agriculture y joue un rôle fondamental durant l'Âge du Fer et à l'époque romaine, et il y a tout lieu de penser qu'elle ne disparaît jamais vraiment. À l'époque carolingienne, l'analyse anthracologique du site d'Augery-deCorrège en Tête-de-Camargue ne fournit encore aucun marqueur direct de (re)mise en culture, mais l'analyse sporopollinique du même site montre déjà une augmen- tation régulière des céréales (Laval, Malléa, 1993; Durand, 1998).

$\mathrm{La}$ conjonction des études environnementales et archéologiques portant sur ce milieu sensible qu'est la plaine alluviale du Bas-Rhône apporte donc un enrichissement mutuel : autour des colonies nouvelles, des terres pouvaient être mises en valeur. Les travaux des environnementalistes n'apportent évidemment pas la preuve qu'elles le furent, mais ils autorisent les archéologues et les historiens à appliquer à ces terres un modèle d'exploitation romain (Leveau, 1993a). Lorsque peu après 46 avant J.-C., Tiberius Claudius Néron installe les soldats libérés de la $\mathrm{VI}^{\mathrm{e}}$ légion, ces colons avaient vocation à devenir des agricolae - c'est-à-dire à exploiter un domaine - plutôt que des marins ou des commerçants. Valable pour la colonie d'Orange, ce modèle l'est pour la colonie d'Arles. Si le rôle du commerce est incontestable et si la colonisation romaine se traduit par la construction de villes, la base de l'économie et l'assise des patrimoines n'en sont pas moins d'abord agricoles et rurales.

\section{HYDROLOGIE ET TERRITOIRE : AVIGNON, VILLE DU RHÔNE}

La relation entre dynamiques fluviales et dynamiques territoriales, définie dans l'introduction de ce « dossier", trouve ici une application. Pour la période chronologique et le secteur fluvial envisagés, elle permet de caractériser une (première?) personnalité régionale dans la structuration de laquelle le fleuve est l'élément essentiel.

Obstacle majeur, dans la topographie de la vallée et à l'échelle de la région, le Rhône a pu générer une frontière d'État. On lui connaît cette fonction à la fin du Moyen Âge quand il sert de limite entre le royaume de France et les territoires pontificaux. Cette situation est alors nouvelle : « au XIII ${ }^{\mathrm{c}}$ siècle le terroir d'Avignon débordait largement le Rhône et s'étendait sur la rive droite jusqu'à Montaut » (Hayez, 1978, p. 23) ; les empiétements de la royauté française font alors du fleuve une frontière, limite naturelle à son pouvoir. Bien évidemment, dans la vie économique régionale, le Rhône n'est pas une coupure, c'est une artère de circulation qui traverse une plaine dont les paysages sont semblables et sur les deux rives de laquelle vivent les mêmes populations. 
Cette situation apparaît parfaitement à l'époque préromaine dans l'épisode déjà cité de la traversée d'Hannibal en 218 avant J.-C., premier exemple historique d'une telle situation. Face aux Carthaginois, qui arrivent de l'ouest, le fleuve fournit aux Volques un point d'appui : abandonnant la défense de la partie occidentale de leur territoire, ils s'installent en rive orientale (gauche) et utilisent le fleuve comme un rempart. Dans ce secteur, durant la phase ultime de la Protohistoire celle qui est documentée par les textes des historiens grecs et latins relatant la conquête romaine - le Rhône ne joue pas le rôle de limite, tout simplement parce que les vastes confédérations qui se forment alors réunissent des tribus assises sur des régions géographiques et que sa vallée en constitue précisément une d'importance. La carte de ces confédérations reste incertaine mais, pour l'espace qui nous intéresse, on y reconnaît bien deux ensembles : à l'ouest, en Languedoc, les Volques et à l'est, en Provence, les Salyens.

Sous le règne de Claude, une époque où la confédération salyenne a disparu, Pomponius Méla écrit que le Rhône se jette (emittitur) entre Volques et Cavares (inter Volcas et Cavaras, II, 79-80). Son objectif est de situer le Rhône et ces peuples les uns par rapport aux autres; dans cette perspective, il n'envisage pas le détail d'une. frontière. L'organisation romaine de la Gaule du Sud en assure l'unité dans la Province de Narbonnaise. Aucun envahisseur ne la menace plus, de sorte que la capacité du Rhône à jouer un rôle de frontière d'État ne présente aucun intérêt. Dans les textes, les Cavares apparaissent au moment où est réalisée cette restructuration territoriale. $\mathrm{Ce}$ ne sont pas des nouveaux venus mais une ethnie qui s'est sans doute fondue dans une confédération (la confédération volque ?). L'administration provinciale romaine favorise l'émergence de cités territoriales organisées autour d'un chef-lieu. C'est alors que naissent des villes axées sur le fleuve, qui disposent de territoires à surface réduite, s'interposant entre les vastes territoires des deux cités héritières des grandes confédérations de l'époque de la conquête, Nîmes des Arécomiques, à l'ouest, et les Voconces, à l'est. Au sud, Arles fait exception, mais l'étendue et l'allongement de son territoire paraissent liés à une commodité administrative consistant à attribuer les terres des Salyens (qui ont disparu) aux deux colonies romaines, Arles et Fréjus, et à Aquae Sextiae, la colonie latine issue du castellum fondé au lendemain de la conquête.
L'urbanisation du Bas-Rhône apparaît remarquable. Deux articles ont fait le point sur les relations d'Arles et d'Avignon avec le fleuve. Cette dernière, l'une des villes nées sur les terres cavares, offre un cas exemplaire. En dehors de ce qu'il apporte sur le thème de ce "dossier ", un des intérêts de la mise au point de D. Carru est de montrer l'importance de la ville à l'époque romaine. Elle incite à restituer à Avignon, la grande méconnue de la vallée du Rhône, des documents dont l'attribution lui a été contestée. Les études épigraphiques de J. Gascou l'ont montré pour le HautEmpire : une inscription, attestant le statut de colonie romaine qui lui est attribué au $\mathrm{II}^{\mathrm{e}} \mathrm{s}$. de notre ère, avait été considérée comme un faux épigraphique fabriqué pour servir le chauvinisme local (Gascou, 1990)! Un autre bloc épigraphe indiquant l'instauration du culte de Drusilla par le futur empereur Claude à la fin du règne de Caligula avait été présumé amené de Lyon par le Rhône (Gascou, 1998) ! En fait, une série de données écrites permet de reconstituer en partie l'évolution juridique d'Avignon au $\mathrm{I}^{\mathrm{cr}} \mathrm{s}$. avant notre ère. Une allusion de César nous apprend que Marseille avait reçu des terres dans ce secteur (César, Guerre civile, I, 35, 4) et une autre, de Cicéron, que les habitants d'Avignon étaient considérés comme fédérés. À l'origine, la ville était en territoire volque si l'on reporte sur une carte l'affirmation de Tite-Live utilisée ci-dessus. Après la défaite de Marseille, elle le redevint selon l'interprétation qu'Hirschfeld donnait de l'inscription gravée sur le socle de la statue de $\mathrm{T}$. Carisius, praetor volcarum (CIL XIII, 1028) (Hirschfeld, 1888, rééd. 1962, p. 346) ${ }^{78}$. Ajoutons que, pas plus que pour Marseille, la faible dimension du territoire n'est la preuve d'une situation économique et politique secondaire. Les changements d'appartenance administrative dont Avignon est l'objet, puis sa promotion à l'ćgal d'Arlcs, sont le témoignage d'une émergence comme "ville du Rhône " plus que comme centre de tel ou tel peuple. Sa personnalité apparaît nettement dans le domaine territorial. Lorsqu'une fraction des Cavares la constitue en chef-lieu de cité romaine (ou lorsque Rome constitue en cité d'Avennio une fraction des Cavares), on ne voit pas pourquoi le territoire de la cité nouvelle ne se serait pas

78. Ce texte a été réexaminé par $M$. Christol et $C$. Goudincau qui se sont intéressés à la fonction de $\mathrm{T}$. Carisius et à sa date, mais pas au problème du territoire (Christol, Goudineau, 1987-1988). 
étendu à la rive droite du fleuve comme l'évêché avant que le roi n'intervienne.

L'histoire de toute la région qui s'étend au sud-est du confluent avec la Durance devra être revue à la lumière de ce qui a été écrit plus haut. On admet qu' " une vaste zone marécageuse s'étirait, dans l'Antiquité, depuis Tarascon jusqu'au Plan d'Orgon " (Barruol, 1975, p. 238) ; elle aurait constitué la limite du territoire cavare par rapport aux Salyens (?) et aurait été reprise pour délimiter les cités d'Arles et d'Avignon. Selon G. Barruol, la région de la Petite Crau aurait été mise en culture à l'époque d'Auguste, irriguée dans sa partie haute, asséchée dans sa partie basse. Son argumentation est fondée sur " le réseau des voies de communication (routes d'Ernaginum-Avennio et Tarusco-Cabellio) et la répartition des grands domaines agricoles "sur lesquels, à l'époque où il écrivait, on ne disposait que de la Forma (Benoit, 1936, p. 196-225) et des premiers travaux sur les centuriations (Chevallier, 1961). Or, depuis, cartes archéologiques et problématiques ont progressé. On est frappé par la densité des villes romaines dotées d'un statut juridique honorifique. Le cas d'Avignon a été souligné. Il est maintenant certain que Glanum a été un moment colonie (Christol, Janon, 1999, p. 79-82). Comme pour Arles, cette prospérité s'appuie sur le développement rural. Pour l'époque romaine, il y a vingt ans déjà, on savait que la toponymie ne pouvait pas fournir la carte des grands domaines gallo-romains. On se rend compte que les villae du Haut-Empire ne correspondent qu'à un moment dans l'histoire du domaine (Pellecuer, à paraître ; Carru et al., à paraître). Surtout, rappelons que l'un des acquis de l'histoire naturelle de la plaine du Rhône est le caractère récent de l'extension du lit majeur du fleuve, luimême un des facteurs importants du développement du marais. On connaît par ailleurs des défluviations de la Durance (Lonchambon, 1996).

L'analyse territoriale ramène donc à une constatation faite à plusieurs reprises. La théorie historique s'est trop appuyée sur l'état de la vallée du Rhône au début du $\mathrm{XIX}^{\mathrm{e}} \mathrm{s}$. : depuis plusieurs siècles, elle était soumise à de graves inondations; la Révolution venait de créer des départements assis sur des limites " naturelles » et donc appuyés sur le fleuve. Les progrès réalisés par les envi- ronnementalistes dans l'histoire (naturelle) des dynamiques rhodaniennes incitent à reprendre tout ce qui été écrit pour la période antique sur l'organisation territoriale de ces régions.

$$
\text { * * }
$$

Le bilan présenté intervient à un moment où l'élargissement de la recherche nécessite un passage de l'échelle locale à l'échelle régionale. Cela est vrai de l'étude sur la plaine d'Arles qui a débuté il y a dix ans sur la vallée des Baux. Les théories en cours sur les moulins de Barbegal, la batellerie arlésienne et les utriculaires se fondaient sur une histoire de l'hydrologie régionale actuellement largement remise en question. Des constatations analogues sont faites sur d'autres secteurs.

Des résultats importants sont attendus des collaborations géoarchéologiques en cours sur la Camargue et sur le secteur amont concerné par la construction de la ligne TGV, ainsi que sur la plaine d'Orange. L'ensemble des données obtenues devrait permettre de faire avancer une question dont on a pu s'étonner qu'elle ait été peu abordée ici, celle des centuriations. Cette question qui, il y a une dizaine d'années, occupait l'essentiel de la recherche sur le paysage, devra être reprise à partir des travaux de terrain réalisés en Languedoc par un certain nombre de chercheurs de l'AFAN dans le cadre d'opérations d'archéologie préventive. Pour la plaine d'Arles et le secteur durancien, le foisonnement des centuriations publiées reflète manifestement l'inachèvement de la recherche. Dans l'attente de sa reprise sur des bases qui ne peuvent plus être exclusivement archéomorphologiques, l'histoire du chenal fluvial permet d'observer un « changement romain» que l'on peut ainsi résumer. Des canaux sont construits par les conquérants pour entrer dans le delta - le canal de Marius - puis, un siècle et demi après, à l'entrée dans la plaine d'Orange, pour contourner une zone difficile. Ces aménagements favorisent l'émergence des villes du Rhône. Certes, à cette époque, ses crues affectent Avignon et Arles. Mais l'image du Rhône antique, qui ressort de l'histoire de cette section de la plaine, est celle d'un fleuve sans doute moins « rebelle et indomptable » que celui qui inspira à Vauban cette expression. 